\title{
NONCOMMUTATIVE QUANTUM MECHANICS VIEWED FROM FEYNMAN FORMALISM
}

\author{
J. LAGES \\ Institut UTINAM, Dynamique des Structures Complexes, UMR du CNRS 6213, \\ Université de Franche-Comté, Campus La Bouloie, 25030 Besançon Cedex, France \\ E-mail: jose.lages@utinam.cnrs.fr \\ A. BERARD, H. MOHRBACH and Y. GRANDATI \\ Laboratoire de Physique Moléculaire et des Collisions, \\ ICPMB, IF CNRS $N^{\circ}$ 2843, Université Paul Verlaine, \\ Institut de Physique, Bd Arago, 57078 Metz Cedex 3, France \\ P. GOSSELIN \\ Institut Fourier, Université de Grenoble I, UMR 5582 CNRS-UJF, \\ UFR de Mathématiques, BP74, 38402 St Martin d'Hères, Cedex France
}

\begin{abstract}
Dyson published in 1990 a proof due to Feynman of the Maxwell equations. This proof is based on the assumption of simple commutation relations between position and velocity. We first study a nonrelativistic particle using Feynman formalism. We show that Poincaré's magnetic angular momentum and Dirac magnetic monopole are the direct consequences of the structure of the $\mathrm{sO}(3)$ Lie algebra in Feynman formalism. Then we show how to extend this formalism to the dual momentum space with the aim of introducing Noncommutative Quantum Mechanics which was recently the subject of a wide range of works from particle physics to condensed matter physics.
\end{abstract}

Keywords: Noncommutative Quantum Mechanics, Feynman formalism, Dirac magnetic monopole, Poincaré momentum, Maxwell's equations, Angular algebra symmetry, Dirac quantization, Berry curvature

\section{Introduction}

Feynman's ideas ${ }^{1}$ were exposed by Dyson in an elegant publication. Initial Feynman's motivation was to develop a quantization procedure without resort to a Lagrangian or a Hamiltonian. Assuming minimal commutation relations between position and velocity and using Newton's second law, Feynman derived the sourceless set of Maxwell's equations which are galileo invariant. The interpretation of the Feynman's derivation of the Maxwell's equations has $\operatorname{aroused}^{2-12}$ a great interest among physicists. In particular Tanimura ${ }^{2}$ has generalized the Feynman's derivation in a Lorentz covariant form with a scalar time evolution parameter. An extension of the Tanimura's approach has been achieved ${ }^{3}$ in using the Hodge duality to derive the two groups of Maxwell's equations with a magnetic monopole in a flat and in a 
curved spaces. In Ref. 4 the descriptions of relativistic and non relativistic particles in an electromagnetic field was studied, whereas in Ref. 5 a dynamical equation for spinning particles was proposed. A rigorous mathematical interpretation of Feynman's derivation connected to the inverse problem for the Poisson dynamic has been formulated in Ref. 6. Also in Refs. 7 and 8 the Feynman's derivation is considered in the frame of the Helmholtz's inverse problem for the calculus of variations. Other works $^{9-11}$ have provided new looks on the Feynman's derivation of the Maxwell's equations. More recently, ${ }^{13}$ some of the authors embedded Feynman's derivation of the Maxwell's equation in the framework of noncommutative geometry. As Feynman's brackets can be interpreted as a deformation of Poisson brackets we showed that the Feynman brackets can be viewed as a generalization of the Moyal brackets defined over the tangent bundle space. ${ }^{13}$

The mathematical foundations of Feynman's formalism is presented in Section 2 and is used to review the Feynman's derivation of the Maxwell's equation in Section 3. It is well known that velocities do not commute in the presence of an electromagnetic field. For this reason the angular algebra symmetry, e.g. the sO(3) symmetry in the Euclidean case, is broken. In Section 4 we show how to restore such a symmetry and we point out in this context the necessity of adding the Poincaré momentum $\mathbf{M}$ to the simple angular momentum $\mathbf{L}$. The direct consequence of this restoration is then the generation of a Dirac magnetic monopole. A natural extension of Feynman's formalism is to consider the dual momentum space. In Section 5] we embed then our work in the natural generalization of Quantum Mechanics involving noncommutative coordinates. This generalization was originally introduced by Snyder ${ }^{14}$ as a short distance regularization to improve the problem of infinite self energies inherent in a Quantum Field Theory. Due to the advent of the renormalization theory this idea was not very popular until Connes ${ }^{15}$ analyzed Yang Mills theories on noncommutative space. More recently a correspondence between a noncommutative gauge theory and a conventional gauge theory was introduced by Seiberg and Witten. ${ }^{16}$ Noncommutative gauge theories were also found as being naturally related to string and M-theory. ${ }^{17}$ Applications of noncommutative theories were also found in condensed matter physics, for instance in the Quantum Hall effect $^{18}$ and in the noncommutative Landau problem, ${ }^{19,20}$ the name of Noncommutative Quantum Mechanics started then to be used. ${ }^{20-23}$ In section 5 we resume our paper $^{24}$ which shows that in our model a quantum particle in a harmonic potential has a behavior similar to a particle in a constant magnetic field $\theta$ in standard quantum mechanics, since a paramagnetic term appears in the Hamiltonian. Moreover the particle acquires an effective dual mass in the same way that an electron moving in a periodic potential in solid state physics. Again the angular algebra symmetry is naturally broken and the restoration of this symmetry gives then a dual Dirac monopole in momentum space field configuration. 


\section{Mathematical foundations of Feynman's formalism}

Let a particle with a mass $m$ and an electrical charge $q$ be described by the vector $\mathbf{x}=\left\{x^{i}\right\}_{i=1, \ldots, N}$ which defines its position on the manifold $\mathcal{M}$. Let the manifold $\mathcal{M}$ be a $N$-dimensional vectorial manifold diffeomorphic to $\mathbb{R}^{N}$. Let $\tau$ be the parameter of the group of diffeomorphisms $\mathcal{G}: \mathbb{R} \times \mathcal{M} \rightarrow \mathcal{M}$ such as $\mathcal{G}(\tau, \mathbf{x})=\mathcal{G}^{\tau} \mathbf{x}=\mathbf{x}(\tau)$. Then taking $\tau$ as the time parameter of our physical system we are able to define a velocity vector $\dot{\mathbf{x}} \in \mathcal{M}$ as $\dot{\mathbf{x}}=\frac{d \mathbf{x}}{d \tau}=\mathcal{G}^{\tau} \mathbf{x}=\left\{\dot{x}^{i}(\tau)\right\}_{i=1, \ldots, N}$. Let $T(\mathcal{M})$ be the tangent bundle space associated with the manifold $\mathcal{M}$, a point on $T(\mathcal{M})$ is described then by a $2 N$ dimensional vector $\boldsymbol{\xi}=\{\mathbf{x}, \dot{\mathbf{x}}\}$. Let $A^{0}(T(\mathcal{M}))=C^{\infty}(T(\mathcal{M}), \mathbb{R})$ be the algebra of differential functions defined on the manifold $T(\mathcal{M})$. We define a Poisson structure on $T(\mathcal{M})$ which is an internal skew-symmetric bilinear multiplicative law on $A^{0}(T(\mathcal{M}))$ denoted $(f, g) \rightarrow[f, g]$ and satisfying the Leibnitz rule

$$
[f, g h]=[f, g] h+[f, h] g
$$

and the Jacobi identity

$$
J(f, g, h)=[f,[g, h]]+[g,[h, f]]+[h,[f, g]]=0 .
$$

The manifold $T(\mathcal{M})$ with such a Poisson structure is called a Poisson manifold. We define a dynamical system on the Poisson manifold $T(\mathcal{M})$ by the following differential equation

$$
\frac{d f}{d \tau}=[f, H]
$$

where $H \in A^{0}(T(\mathcal{M}))$ is the Hamiltonian of the dynamical system.

With such definitions we derive the following important relations for functions belonging to $A^{0}(T(\mathcal{M}))$

$$
\begin{aligned}
{[f(\boldsymbol{\xi}), h(\boldsymbol{\xi})] } & =\{f(\boldsymbol{\xi}), h(\boldsymbol{\xi})\} \\
& +\left[x^{i}, x^{j}\right] \frac{\partial f(\boldsymbol{\xi})}{\partial x^{i}} \frac{\partial h(\boldsymbol{\xi})}{\partial x^{j}}+\left[\dot{x}^{i}, \dot{x}^{j}\right] \frac{\partial f(\boldsymbol{\xi})}{\partial \dot{x}^{i}} \frac{\partial h(\boldsymbol{\xi})}{\partial \dot{x}^{j}},
\end{aligned}
$$

where we have introduced Poisson-like brackets defined by

$$
\{f(\boldsymbol{\xi}), g(\boldsymbol{\xi})\}=\left[x^{i}, \dot{x}^{j}\right]\left(\frac{\partial f(\boldsymbol{\xi})}{\partial x^{i}} \frac{\partial h(\boldsymbol{\xi})}{\partial \dot{x}^{j}}-\frac{\partial f(\boldsymbol{\xi})}{\partial \dot{x}^{i}} \frac{\partial h(\boldsymbol{\xi})}{\partial x^{j}}\right) .
$$

We can see the relation (4) as the simple deformation of the Poisson-like brackets introduced in (5). It is obvious that the tensors $\left[x^{i}, x^{j}\right]$ and $\left[\dot{x}^{i}, \dot{x}^{j}\right]$ are skew symmetric.. We introduce then the following notations

$$
\left[x^{i}, x^{j}\right]=\frac{q}{m^{2}} \theta^{i j}(\boldsymbol{\xi}), \quad\left[x^{i}, \dot{x}^{j}\right]=\frac{1}{m} g^{i j}(\boldsymbol{\xi}), \quad\left[\dot{x}^{i}, \dot{x}^{j}\right]=\frac{q}{m^{2}} F^{i j}(\boldsymbol{\xi})
$$

where $g^{i j}(\boldsymbol{\xi})$ is the $N \times N$ metric tensor, and where $\theta^{i j}(\boldsymbol{\xi})$ and $F^{i j}(\boldsymbol{\xi})$ are two $N \times N$ skew symmetric tensors, $F^{i j}(\boldsymbol{\xi})$ being related to the electromagnetic tensor introduced in a preceding paper. ${ }^{25}$ 


\section{Maxwell's equations}

In a three dimensional flat space we have $g^{i j}(\mathbf{x})=\delta^{i j}$ and the Hamiltonian of the Poisson structure reads then

$$
H=\frac{1}{2} m \dot{x}^{i} \dot{x}_{i}+f(\mathbf{x})
$$

The Jacobi identity (2) involving position and velocity components

$$
J\left(x^{i}, \dot{x}^{j}, \dot{x}^{k}\right) \propto \frac{\partial F^{j k}(\boldsymbol{\xi})}{\partial \dot{x}^{i}}=0 .
$$

shows that the gauge curvature is velocity independent, $F^{i j}(\boldsymbol{\xi}) \equiv F^{i j}(\mathbf{x})$. From the Jacobi identity (2) involving only velocities components we derive the Bianchi equation

$$
J\left(\dot{x}^{i}, \dot{x}^{j}, \dot{x}^{k}\right) \propto \varepsilon^{k}{ }_{j i} \frac{\partial F^{i j}(\mathbf{x})}{\partial x^{k}}=0
$$

which, if we set $F^{i j}(\mathbf{x})=\varepsilon^{j i}{ }_{k} B^{k}(\mathbf{x})$, gives the following Maxwell's equation

$$
\nabla \cdot \mathbf{B}=0 .
$$

Now using the dynamical equation (3) we obtain the following equation of motion

$$
m \ddot{x}^{i}=m\left[\dot{x}^{i}, H\right]=q F^{i j}(\mathbf{x}) \dot{x}_{j}+q E^{i}(\mathbf{x})
$$

where

$$
q E^{i}(\mathbf{x})=-\frac{\partial f(\mathbf{x})}{\partial x_{i}}
$$

We have then a particle of mass $m$ and electrical charge $q$ moving in flat space where a magnetostatic and an electrostatic external field are present. We are able now to derive the other Maxwell's equation of the first group. With the dynamical equation (3) we express the time derivative of the magnetic field

$$
\frac{d B^{i}}{d t}=\frac{1}{2} \varepsilon^{i}{ }_{j k}\left[F^{j k}, H\right]=\frac{m^{2}}{2 q} \varepsilon^{i}{ }_{j k}\left[\left[\dot{x}^{j}, \dot{x}^{k}\right], H\right]
$$

and we use the Jacobi identy (2) to rewrite the last term of the last equation. After some calculus we obtain

$$
\frac{d B^{i}}{d t}=-\dot{x}^{i} \nabla \cdot \mathbf{B}+\frac{\partial B^{i}}{\partial x_{j}} \dot{x}_{j}+\varepsilon^{i}{ }_{j k} \frac{\partial E^{j}}{\partial x_{k}}
$$

which using (9) gives the second Maxwell's equation

$$
\frac{\partial \mathbf{B}}{\partial t}-\nabla \times \mathbf{E}=\mathbf{0}
$$

for static fields and electric fields deriving from any potential $f(\mathbf{x})$ (11).

As the two other Maxwell's equations are not Galileo invariant they cannot be deduced from the formalism and can be merely seen as a definition of the charge density and the current density. Nevertheless, as shown in Ref. 13 the complete set of the Maxwell's equations can be deduced in the relativistic generalization. 


\section{4. sO(3) algebra and Poincaré momentum}

One of the most important symmetry in physics is naturally the spherical symmetry corresponding to the isotropy of the physical space. This symmetry is related to the $\mathrm{sO}(3)$ algebra. In the following we show that this symmetry is broken when an electromagnetic field is applied. In order to study the symmetry breaking of the $\mathrm{sO}(3)$ algebra we use the usual angular momentum $L^{i}=m \varepsilon^{i}{ }_{j k} x^{j} \dot{x}^{k}$ which is a constant of motion in absence of gauge field. In fact, no electromagnetic field implies $F^{i j}(\mathbf{x})=\left[\dot{x}^{i}, \dot{x}^{j}\right]=0$, and the expression of the $\mathrm{sO}(3)$ Lie algebra with our brackets (4) gives then the standard algebra defined in terms of the Poisson brackets (5)

$$
\left\{\begin{array}{l}
{\left[x^{i}, L^{j}\right]=\left\{x^{i}, L^{j}\right\}=\varepsilon^{i j}{ }_{k} x^{k},} \\
{\left[\dot{x}^{i}, L^{j}\right]=\left\{\dot{x}^{i}, L^{j}\right\}=\varepsilon^{i j}{ }_{k} \dot{x}^{k},} \\
{\left[L^{i}, L^{j}\right]=\left\{L^{i}, L^{j}\right\}=\varepsilon^{i j}{ }_{k} L^{k} .}
\end{array}\right.
$$

When the electromagnetic field is turned on this algebra is broken in the following manner

$$
\left\{\begin{aligned}
{\left[x^{i}, L^{j}\right] } & =\left\{x^{i}, L^{j}\right\}=\varepsilon^{i j}{ }_{k} x^{k}, \\
{\left[\dot{x}^{i}, L^{j}\right] } & =\left\{\dot{x}^{i}, L^{j}\right\}+\frac{q}{m} \varepsilon^{j}{ }_{k l} x^{k} F^{i l}(\mathbf{x}) \\
& =\varepsilon^{i j}{ }_{k} \dot{x}^{k}+\frac{q}{m} \varepsilon^{j}{ }_{k l} x^{k} F^{i l}(\mathbf{x}), \\
{\left[L^{i}, L^{j}\right] } & =\left\{L^{i}, L^{j}\right\}+q \varepsilon^{i}{ }_{k l} \varepsilon^{j}{ }_{m s} x^{k} x^{m} F^{l s}(\mathbf{x}) \\
& =\varepsilon^{i j}{ }_{k} L^{k}+q \varepsilon^{i}{ }_{k l} \varepsilon^{j}{ }_{m s} x^{k} x^{m} F^{l s}(\mathbf{x}) .
\end{aligned}\right.
$$

In order to restore the $\mathrm{sO}(3)$ algebra we introduce a new angular momentum $M^{i}(\boldsymbol{\xi})$ which is a priori position and velocity dependent. We consider then the following transformation law

$$
L^{i}(\boldsymbol{\xi}) \rightarrow \mathcal{L}^{i}(\boldsymbol{\xi})=L^{i}(\boldsymbol{\xi})+M^{i}(\boldsymbol{\xi}),
$$

and we require that this new angular momentum $\mathcal{L}^{i}$ verifies the usual $\mathrm{sO}(3)$ algebra

$$
\left\{\begin{array}{l}
{\left[x^{i}, \mathcal{L}^{j}\right]=\left\{x^{i}, \mathcal{L}^{j}\right\}=\varepsilon^{i j}{ }_{k} x^{k},} \\
{\left[\dot{x}^{i}, \mathcal{L}^{j}\right]=\left\{\dot{x}^{i}, \mathcal{L}^{j}\right\}=\varepsilon^{i j}{ }_{k} \dot{x}^{k},} \\
{\left[\mathcal{L}^{i}, \mathcal{L}^{j}\right]=\left\{\mathcal{L}^{i}, \mathcal{L}^{j}\right\}=\varepsilon^{i j}{ }_{k} \mathcal{L}^{k} .}
\end{array}\right.
$$

These equations (18) gives then three constrains on the expression of the angular momentum $\mathcal{L}^{i}$. From the first relation in (18) we easily deduce that $M^{i}$ is velocity independent $M^{i}(\boldsymbol{\xi})=M^{i}(\mathbf{x})$, from the second relation we obtain

$$
\left[\dot{x}^{i}, M^{j}\right]=-\frac{1}{m} \frac{\partial M^{j}(\mathbf{x})}{\partial x_{i}}=-\frac{q}{m} \varepsilon_{k l}^{j} x^{k} F^{i l}(\mathbf{x})
$$

and finally the third relation gives

$$
M^{i}=\frac{1}{2} q \varepsilon_{j k l} x^{i} x^{k} F^{j l}(\mathbf{x})=-q(\mathbf{x} \cdot \mathbf{B}) x^{i} .
$$

Equations (19) and (20) are compatible only if the magnetic field $\mathbf{B}$ is the Dirac magnetic monopole field

$$
\mathbf{B}=\frac{g}{4 \pi} \frac{\mathbf{x}}{\|\mathbf{x}\|^{3}} .
$$


The vector $\mathbf{M}$ allowing us to restore the $\mathrm{sO}(3)$ symmetry (18) is then the Poincaré momentum ${ }^{26}$

$$
\mathbf{M}=-\frac{q g}{4 \pi} \frac{\mathbf{x}}{\|\mathbf{x}\|} .
$$

already found in a preceding paper ${ }^{27} \cdot{ }^{28}$ The total angular momentum is then

$$
\mathcal{L}=\mathbf{L}-\frac{q g}{4 \pi} \frac{\mathbf{x}}{\|\mathbf{x}\|}
$$

This expression was initially found by Poincaré in a different context. ${ }^{26}$ Actually he was looking for a new angular momentum that would be a constant of motion. In our framework this property is trivially verified by using the dynamical relation (3). This procedure of symmetry restoration has also been performed for Lorentz algebra in a curved space. ${ }^{13}$ An other generalization of this formalism can be find in a recent interesting work where the study of the Lorentz generators in $\mathrm{N}$-dimensional Minkowski space is proposed..$^{29,30}$

Let us now discuss an important point. As the Dirac magnetic monopole is located at the origin we have

$$
J\left(\dot{x}^{i}, \dot{x}^{j}, \dot{x}^{k}\right)=\nabla \cdot \mathbf{B}=g \delta^{3}(\mathbf{x}) .
$$

The preservation of the $\mathrm{sO}(3)$ symmetry in the presence of a gauge field is then incompatible with the requirement of the Jacobi identity at the origin of the coordinates and we have to exclude the origin from the manifold $\mathcal{M}$. As the Jacobi identity is the infinitesimal statement of associativity in the composition law of the translation group, ${ }^{31}$ the breakdown of the Jacobi identity (23) when $\nabla \cdot \mathbf{B} \neq 0$ implies that finite translations do not associate. In usual quantum mechanics nonassociativity between operators acting on the Hilbert space can not be tolerate, one has to use the Dirac's quantization procedure to save associativity (23).

In order to consider quantum mechanics within our framework we have to quantify as usual the total angular momentum $\mathcal{L}$. Considering the rest frame of the particle we have the following Dirac quantization

$$
\frac{g q}{4 \pi}=\frac{n}{2} \hbar
$$

\section{Noncommutative Quantum Mechanics}

Let now the momentum vector $\mathbf{p}$ replace the velocity vector $\dot{\mathbf{x}}$ in the Feynman formalism presented in Sec. 2. Consider a quantum particle of mass $m$ whose coordinates satisfy the deformed Heisenberg algebra

$$
\left[x^{i}, x^{j}\right]=i \hbar q_{\theta} \theta^{i j}(\mathbf{x}, \mathbf{p}), \quad\left[x^{i}, p^{j}\right]=i \hbar \delta^{i j}, \quad\left[p^{i}, p^{j}\right]=0,
$$

where $\theta$ is a field which is a priori position and momentum dependent and $q_{\theta}$ is a charge characterizing the intensity of the interaction of the particle and the $\theta$ field. The commutation of the momentum implies that there is no external magnetic field. It is well known that these commutation relations can be obtained from the 
deformation of the Poisson algebra of classical observable with a provided WeylWigner-Moyal product ${ }^{32}$ expanded at the first order in $\theta$.

\subsection{Jacobi identities}

The Jacobi identity $J\left(p^{i}, x^{j}, x^{k}\right)=0$ implies the important property that the $\theta$ field is position independent $\theta^{j k}=\theta^{j k}(\mathbf{p})$. Then one can see the $\theta$ field like a dual of a magnetic field and $q_{\theta}$ like a dual of an electric charge. The fact that the field is homogeneous in space is an essential property for the vacuum. In addition, one easily see that a particle in this field moves freely, that is, the vacuum field does not act on the motion of the particle in the absence of an external potential. The effect of the $\theta$ field is manifest only in presence of a position dependent potential. To look further at the properties of the $\theta$ field consider the other Jacobi identity $J\left(x^{i}, x^{j}, x^{k}\right)=0$ giving the equation of motion of the field

$$
\frac{\partial \theta^{j k}(\mathbf{p})}{\partial p^{i}}+\frac{\partial \theta^{k i}(\mathbf{p})}{\partial p^{j}}+\frac{\partial \theta^{i j}(\mathbf{p})}{\partial p^{k}}=0
$$

which is the dual equation of the Maxwell equation $\nabla \cdot \mathbf{B}=0$. As we will see later, equation (25) is not satisfied in the presence of a monopole and this will have important consequences.

\subsection{Position transformation}

Now consider the position transformation $X^{i}=x^{i}+q_{\theta} a_{\theta}^{i}(\mathbf{x}, \mathbf{p})$, where $a_{\theta}$ is a priori position and momentum dependent, that restores the usual canonical Heisenberg algebra

$$
\left[X^{i}, X^{j}\right]=0, \quad\left[X^{i}, p^{j}\right]=i \hbar \delta^{i j}, \quad\left[p^{i}, p^{j}\right]=0 .
$$

The second commutation relation implies that $a_{\theta}$ is position independent, while the commutation relation of the positions leads to the following expression of $\theta$ in terms of the dual gauge field $a_{\theta}$

$$
\theta^{i j}(\mathbf{p})=\frac{\partial a_{\theta}^{i}(\mathbf{p})}{\partial p^{j}}-\frac{\partial a_{\theta}^{j}(\mathbf{p})}{\partial p^{i}}
$$

which is dual to the standard electromagnetic relation in position space.

\subsection{Field Properties}

In order to examine more in detail the properties of this new field, let us consider initially the case of a constant field what is usual in noncommutative quantum mechanics. In the case of an harmonic oscillator expressed in terms of the original $\{\mathbf{x}, \mathbf{p}\}$ coordinates the Hamiltonian reads

$$
H_{\theta}(\mathbf{x}, \mathbf{p})=\frac{\mathbf{p}^{2}}{2}+\frac{k}{2} \mathbf{x}^{2}
$$


from which we have $p^{i}=m \dot{x}^{i}-k q_{\theta} \theta^{i j} x_{j}, \dot{p}^{i}=-k x^{i}$ and the equation of motion $m \ddot{x}^{i}=k q_{\theta} \theta^{i j} \dot{x}_{j}-k x^{i}$ which corresponds formally to a particle in a harmonic oscillator submitted to an external constant magnetic field. From equation (26) we deduce that $a_{\theta}^{i}(\mathbf{p})=q_{\theta} \theta^{i j} p_{j}$, so $X^{i}=x^{i}+\frac{1}{2} q_{\theta} \theta^{i j} p_{j}$, and the Hamiltonian can then be written

$$
H_{\theta}(\mathbf{X}, \mathbf{p})=\frac{\left(m_{*}^{-1}\right)^{i j} p_{i} p_{j}}{2}+\frac{k}{2} \mathbf{X}^{2}-k \frac{q_{\theta}}{2 m} \boldsymbol{\Theta} \cdot \mathcal{L},
$$

with $\theta^{i j}=\varepsilon^{i j k} \Theta_{k}, \mathcal{L}^{i}(\mathbf{X}, \mathbf{p})=\frac{1}{2} \varepsilon^{i}{ }_{j k}\left(X^{j} p^{k}+p^{k} X^{j}\right)$ and $\sigma^{i j}=\delta^{i j} \Theta^{2}-\Theta^{i} \Theta^{j}$, the dual tensor of the Maxwell constraint tensor. Note that the interaction with the field $\theta$ is due to the presence of the position dependent harmonic potential and leads to a dual paramagnetic interaction which could be experimentally observable. Like an electron in the effective periodic potential of ions, the particle in the $\theta$ field acquires an effective mass tensor $m_{*}^{i j}=m\left(\delta^{i j}+\frac{\hbar^{2} k q_{\theta}^{2}}{4} \sigma^{i j}\right)^{-1}$ which breaks the homogeneity of space. This strong analogy with the vacuum of the solid state leads us to consider this field as a property of the vacuum.

\subsection{Angular momentum}

Consider now the problem of angular momentum. It is obvious that the angular momentum expressed according to the canonical coordinates satisfies the angular momentum algebra however it is not conserved

$$
\frac{d}{d t} \mathcal{L}(\mathbf{X}, \mathbf{p})=k q_{\theta} \mathcal{L} \wedge \boldsymbol{\Theta} .
$$

In the original $\{x, p\}$ space the usual angular momentum $L^{i}(\mathbf{x}, \mathbf{p})=\varepsilon^{i}{ }_{j k} x^{j} p^{k}$, does not satisfy this algebra. So it seems that there are no rotation generators in the $\{x, p\}$ space. We will now prove that a true angular momentum can be defined only if $\theta$ is a non constant field. From the definition of the angular momentum we deduce the following commutation relations

$$
\begin{gathered}
{\left[x^{i}, L^{j}\right]=i \hbar \varepsilon^{i j k} x_{k}+i \hbar q_{\theta} \varepsilon^{j}{ }_{k l} p^{l} \theta^{i k}(\mathbf{p}), \quad\left[p^{i}, L^{j}\right]=i \hbar \varepsilon^{i j k} p_{k},} \\
{\left[L^{i}, L^{j}\right]=i \hbar \varepsilon^{i j}{ }_{k} L^{k}+i \hbar q_{\theta} \varepsilon^{i}{ }_{k l} \varepsilon^{j}{ }_{m n} p^{l} p^{n} \theta^{k m}(\mathbf{p}),}
\end{gathered}
$$

showing in particular that the $\mathrm{sO}(3)$ Algebra is broken. To restore the angular momentum algebra consider the transformation law

$$
L^{i} \rightarrow \mathbb{L}^{i}=L^{i}+M_{\theta}^{i}(\mathbf{x}, \mathbf{p}),
$$

and require the usual algebra

$$
\left[x^{i}, \mathbb{L}^{j}\right]=i \hbar \varepsilon^{i j k} x_{k}, \quad\left[p^{i}, \mathbb{L}^{j}\right]=i \hbar \varepsilon^{i j k} p_{k}, \quad\left[\mathbb{L}^{i}, \mathbb{L}^{j}\right]=i \hbar \varepsilon^{i j k} \mathbb{L}_{k} .
$$

The second equation in (33) implies the position independent property $M_{\theta}^{j}(\mathbf{x}, \mathbf{p})=$ $M_{\theta}^{j}(\mathbf{p})$, while the third equation leads to

$$
M_{\theta}^{i}(\mathbf{p})=\frac{1}{2} q_{\theta} \varepsilon_{j k l} p^{i} p^{l} \theta^{k j}(\mathbf{p}) .
$$


Using this equation we rewrite the third equation in (33) and we obtain a dual Dirac monopole ${ }^{33}$ defined in momentum space

$$
\Theta(\mathbf{p})=\frac{g_{\theta}}{4 \pi} \frac{\mathbf{p}}{\|\mathbf{p}\|^{3}} .
$$

We have introduced the dual magnetic charge $g_{\theta}$ associated to the $\Theta$ field. Consequently we have

$$
\mathbf{M}_{\theta}(\mathbf{p})=-\frac{q_{\theta} g_{\theta}}{4 \pi} \frac{\mathbf{p}}{\|\mathbf{p}\|}
$$

which is the dual of the famous Poincaré momentum introduced in positions space. ${ }^{25,26}$ Then the generalized angular momentum

$$
\mathbb{L}=\mathbf{r} \wedge \mathbf{p}-\frac{q_{\theta} g_{\theta}}{4 \pi} \frac{\mathbf{p}}{\|\mathbf{p}\|} .
$$

is a genuine angular momentum satisfying the usual algebra. It is the summation of the angular momentum of the particle and of the dual monopole field. One can check that it is a conserved quantity. It is interesting to note that the use of this formalism with these coordinates had been made also for massless particles in the context of anyons. ${ }^{34}$

The duality between the monopole in momentum space and the Dirac monopole is due to the symmetry of the commutation relations in noncommutative quantum mechanics where $\left[x^{i}, x^{j}\right]=i \hbar q_{\theta} \varepsilon^{i j k} \Theta_{k}(\mathbf{p})$ and the usual quantum mechanics in a magnetic field where $\left[v^{i}, v^{j}\right]=i \hbar q \varepsilon^{i j k} B_{k}(\mathbf{x})$. Therefore the two gauge fields $\Theta(\mathbf{p})$ and $B(\mathbf{x})$ are dual to each other. Note that in the presence of the dual monopole the Jacobi identity fails

$$
J\left(p^{i}, x^{j}, x^{k}\right)=-q_{\theta} \hbar^{2} \frac{\partial \Theta^{i}(\mathbf{p})}{\partial p_{i}}=-4 \pi q_{\theta} \hbar^{2} g_{\theta} \delta^{3}(\mathbf{p}) \neq 0 .
$$

This term is responsible for the violation of the associativity which is only restored if the following quantification equation is satisfied

$$
\int d^{3} p \frac{\partial \Theta^{i}}{\partial p_{i}}=\frac{2 \pi n \hbar}{q_{\theta}}
$$

leading to $q_{\theta} g_{\theta}=\frac{n \hbar}{2}$, in complete analogy with Dirac's quantization. ${ }^{31}$

\subsection{Physical realization}

A physical realization of our theory was found very recently in the context of the anomalous Hall effect in a ferromagnetic crystal. ${ }^{35}$ The main point is the consideration of the Berry phase ${ }^{36} a_{n}^{\mu}(\mathbf{k})=i\left\langle u_{n \mathbf{k}}\left|d_{k}\right| u_{n \mathbf{k}}\right\rangle$ where the wave function $u_{n \mathbf{k}}$ are the periodic part of the Bloch waves. In their work, the authors introduced a gauge covariant position operator of the wave packet associated to an electron in the $n$ band $x^{\mu}=i \frac{\partial}{\partial k_{\mu}}-a_{n}^{\mu}(\mathbf{k})$, whose commutator is given by

$$
\left[x^{\mu}, x^{\nu}\right]=\frac{\partial a_{n}^{\nu}(\mathbf{k})}{\partial k^{\mu}}-\frac{\partial a_{n}^{\mu}(\mathbf{k})}{\partial k^{\nu}}=-i F^{\mu \nu}(\mathbf{k})
$$


where $F^{\mu \nu}(\mathbf{k})$ is the Berry curvature in momentum space.

The connection with our noncommutative quantum mechanics theory is then clearly apparent. The $\theta(\mathbf{p})$ field corresponds to the Berry curvature $F(k)$ and $a_{\theta}(\mathbf{p})$ is associated to the Berry phase $a_{n}(k)$. This shows that physical situations with a Berry phase living in momentum space could be expressed in the context of a noncommutative quantum mechanics. It is essential to mention that the monopole in momentum space, that we deduced from general symmetry considerations applied to the noncommutative quantum mechanics was highlighted in very beautiful experiments of Fang et al. ${ }^{35}$

\section{Conclusion}

Starting from the derivation of Maxwell's equations we reviewed the Feynman formalism. The angular algebra symmetry is naturally broken in the presence of a magnetic field, we showed within the framework of the Feynman formalism how to restore this symmetry. The restoration generates then a Dirac magnetic monopole and implies in addition to the usual angular momentum an associated Poincaré momentum. In Ref. 13 this restoration has been performed also in curved space and a direct application to gravitoelectromagnetism has been given.

Going from the tangent bundle space to the cotangent bundle space and requiring the restoration of the Heisenberg algebra, we have shown that Noncommutative Quantum Mechanics can be viewed from Feynman formalism. In order to maintain the $\mathrm{sO}(3)$ algebra a dual monopole in momentum space is generated. This monopole is responsible for the violation of the Jacobi identity and implies the non associativity of the law of addition of the momentum. To restore associativity a Dirac's quantization of the dual charges is necessary. As a natural physical realization of our theory we can see the $\theta(\mathbf{p})$ field like a Berry curvature associated to a Berry phase expressed in momentum space. The monopole in momentum space predicted by our generalization of noncommutative quantum mechanics was found recently 35 in condensed matter physics experiments.

\section{References}

1. F. Dyson, Am. J. Phys. 58, p. 209 (1990).

2. S. Tanimura, Ann. Phys. 220, p. 229 (1992).

3. A. Bérard, Y. Grandati and H. Mohrbach, Jour. Math. Phys. 40, p. 3732 (1999).

4. C. R. Lee, Phys. Lett. A 148, p. 146 (1990).

5. C. H. Chou, Phys. Lett. B 323, p. 147 (1994).

6. J. F. Carinena, L. A. Ibort, G. Marmo and A. Stern, Phys. Rep. 263, p. 153 (1995).

7. S. A. Hojman and L. C. Shepley, J. Math. Phys. 32, p. 142 (1991).

8. R. J. Hughes, Am. Jour. Phys. 60, p. 301 (1992).

9. M. Montesinos and A. Perez-Lorentzana, Int. Jour. Theor. Phys. 38, p. 901 (1999).

10. P. Singh and N. Dadhich, Mod. Phys. Lett. A 16, p. 83 (2001).

11. Z. K. Siligadze, Annales Fond. Broglie 27, p. 241 (2002).

12. A. Boulahoual and M. B. Sedra, J. Math. Phys. 445888 (2003)

13. A. Bérard, J. Lages and H. Mohrbach, Eur. Phys. J. C 35, p. 373 (2004). 
14. H. Snyder, Phys. Rev. 71, p. 38 (1947).

15. A. Connes, Noncommutative Geometry (Academic press, San Diego, 1994).

16. N. Seiberg and E. Witten, J. High Energy Phys. 09, p. 32 (1999).

17. A. Konechny and A. Schwarz, Phys. Rep. 360, p. 353 (2002).

18. J. Bellissard, H. Schulz-Baldes and A. van Elst, J. Math. Phys. 35, p. 5373 (1994).

19. G. Dunne, R. Jackiw and C. Trugenberg, Phys. Rev. D 41, p. 661 (1990).

20. C. Duval and P. Horvathy, J. Phys. A 34, p. 10097 (2001).

21. J. Gamboa, F. Méndez, M. Loewe and J. C. Rojas, Mod. Phys. Lett. A 16, p. 2075 (2001).

22. V. P. Nair and A. P. Polychronakos, Phys. Lett. B 505, p. 267 (2001).

23. I. Cortese, J. A. Garcia, Int. Jour. Geom. Meth. Mod. Phys. 4, p. 789 - 805 (2007); I. Cortese, J. A. Garcia, Phys. Lett. A 358 327-333 (2006)

24. A. Bérard and H. Mohrbach, Phys. Rev. D 69, p. 127701 (2004).

25. A. Bérard, H. Mohrbach and P. Gosselin, Int. Jour. Theor. Phys. 39, p. 1051 (2000).

26. H. Poincaré, C. R. Acad. Sci. Paris 123, p. 530 (1896).

27. A. Bérard, Y. Grandati and H. Mohrbach, Phys. Lett. A 254, p. 133 (1999).

28. A. Bérard and H. Mohrbach, Int. Jour. Theor. Phys. 39, p. 2633 (2000).

29. M. Land, hep-th/0603055, (2006).

30. M. Land, hep-th/0606046, (2006).

31. R. Jackiw, Phys. Rev. Lett. 54, p. 159 (1985).

32. J. E. Moyal, Proc. Cam. Phil. Soc. 45, p. 99 (1949).

33. P. A. M. Dirac, Proc. R. Soc. A 113, p. 60 (1931).

34. J. L. Cortes, M. S. Plyushchay, Int. J. Mod. Phys. A 11, p. 3331-3362 (1996); P. Horvathy, M. S. Plyushchay, JHEP 0206:033 (2002); P. Horvathy, M. S. Plyushchay, Phys. Lett. B 595, 547-555 (2004); P. Horvathy, M. S. Plyushchay, Nucl. Phys. B 714, 269-291 (2005).

35. Z. Fang, N. Nagaosa, K. S. Takahashi, A. Asamitsu, R. Mathieu, T. Ogasawara, H. Yamada, M. Kawasaki, Y. Tokura, and K. Terakura, Science 302, p. 92 (2003).

36. M. V. Berry, Proc. R. Soc. London A 392, p. 45 (1984). 